\title{
Self-Triggered Model Predictive Control for Network Scheduling and Control ${ }^{\star}$
}

\author{
Erik Henriksson* Daniel E. Quevedo** Henrik Sandberg* \\ Karl Henrik Johansson* \\ * ACCESS Linnaeus Centre, School of Electrical Engineering, Royal \\ Institute of Technology, 10044 Stockholm, Sweden. \\ e-mail: \{erike02, hsan, kallej\}@ee.kth.se \\ ** School of Electrical Engineering and Computer Science, The \\ University of Newcastle, NSW 2308, Australia. \\ e-mail: dquevedo@ieee.org
}

\begin{abstract}
Herein we present an algorithm for controlling LTI processes using an adaptive sampling interval where the controller at every sampling instant not only computes the new control command but also decides the time interval to the next sample. The approach relies on MPC where the cost function depends on the control performance as well as the cost for sampling. The paper presents a method for synthesizing such a predictive controller and gives explicit conditions for when it is stabilizing. Further it is shown that the optimization problem may be solved off-line and that the controller may be implemented as a lookup table of state feedback gains. The paper is concluded with a numerical example.
\end{abstract}

Keywords: Predictive Control; Process Control; Stability; Networked Control System.

\section{INTRODUCTION}

Wireless sensing and control systems have received increased attention in the process industry over the last years. Emerging technologies in low-power wake-up radio enables engineering of a new type of industrial automation systems where sensors, controllers and actuators communicate over a wireless channel. This introduction of a wireless medium gives rise to new challenges which need to be handled (Willig [2008]). The aim of this paper is to address the problem of how the medium access to the wireless channel could be divided between different loops, taking the process dynamics into consideration. The approach taken is to investigate possibilities to adaptively choose the sampling period of loops, in order to reduce the amount of generated traffic.

Consider the networked control system in Fig. 1, which shows how the sensors and the controller are connected through a wireless network. The wireless network is controlled by a Network Manager which allocates medium access to the different sensors and requests information from them. These requests are in turn generated by the controller which, in addition to computing the appropriate control action, dynamically determines the time of the next sample by a self-triggering approach. In doing so the controller gives different attention to different loops depending on their state, while trying to communicate as seldom as possible. To achieve this the controller must, for every loop, trade control performance against inter sampling time and give a quantitative measure of the resulting performance. As this is the main property of the controller, we will in the following consider a single-loop system and focus on the communication contra control

\footnotetext{
* This work was supported by the Swedish Governmental Agency for Innovation Systems through the WiComPi project, the Swedish Research Council under Grants 2007-6350 and 2009-4565, the Swedish Foundation for Strategic Research under the ICT- $\Psi$ project, and the Knut and Alice Wallenberg Foundation.
}

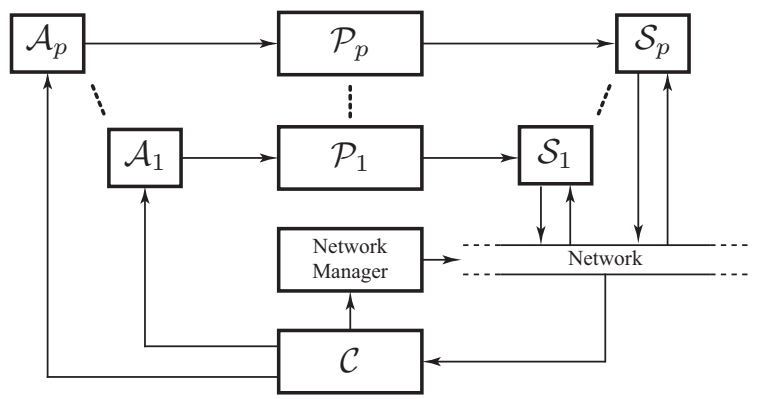

Fig. 1. Actuators $\mathcal{A}$ and processes $\mathcal{P}$ are wired to the controller $\mathcal{C}$ while the sensors $\mathcal{S}$ communicate over the network, which in turn is coordinated by $\mathcal{C}$ via the network manager.

performance trade-off. If the controller works properly it will allow us to maintain performance while reducing the traffic in the network.

The main contribution of the paper is to show that such a self-triggering controller can be derived using a receding horizon control formulation where the predicted cost is used to jointly determine what control signal to be applied as well as the time of the next sampling instant. Using this formulation we can guarantee a minimum and a maximum time between samples.

The development of control strategies for wireless automation has become a large area of research in which, up until recently, most efforts have been made under the assumption of periodic communication (Antsaklis and Baillieul [2007]). However the idea of adaptive sampling is receiving increased attention. The efforts within this area may coarsely be divided into the two paradigms event- and self-triggered control. In event-triggered control (Årzén [1999]; Åström and Bernhardsson [1999]; Tabuada [2007]; Heemels et al. [2008]) the sensor continuously monitors the process state and generates a sample when the state 
violates some predefined condition. Self-triggered control (Velasco et al. [2003]; Wang and Lemmon [2009]; Anta and Tabuada [2010]; Mazo Jr. et al. [2010]) utilizes a model of the system to predict when a new sample needs to be taken in order to fulfill some condition, chosen by design. The advantage of event- over self-triggered control is that the continuous monitoring of the state guarantees that a sample will be drawn as soon the design condition is violated and we may directly apply the appropriate control action. The self-triggered controller will instead, as periodically sampled controllers, operate in open-loop between samples. This could potentially be a problem as changes to the process between samples cannot be measured or attenuated. This problem may however be avoided by good choices of the inter sampling times. The advantage of selfover event-triggered control which we utilize here is that the transmission time of sensor packets is known a-priori and hence we may schedule them, enabling sensors and transmitters to be put to sleep in between samples and thereby save energy.

The use of predictive control is gaining popularity within the networked control community (Casavola et al. [2006]; Tang and de Silva [2006]; Liu et al. [2006]; Zhao et al. [2008]). In an adaptive sampling context it has previously been used in (Eqtami et al. [2010]; Bernardini and Bemporad [2011]) for the case of event-based sampling and in (Liu et al. [2010]) for the case of asynchronous measurements. The approach to jointly optimize over the inter sampling time and the control performance as done in this paper is however to the best of the authors knowledge new.

The outline of the paper is as follows. In Section 2 the problem is defined and outlined as a receding horizon control problem. Section 3 presents the open-loop optimal control problem, to be solved by the receding horizon controller, together with its optimal solution. Section 4 presents the receding horizon control algorithm in further detail and gives conditions for when it is stabilizing. The proposed method is explained and evaluated on a simulated numerical example in Section 5. Concluding discussions are made in Section 6.

Notation: We have tried to use standard notation in the paper. Let $\mathbb{N}^{+}=\mathbb{N} \backslash\{0\}$. By $\hat{x}\left(k^{\prime} \mid k\right)$ we denote an estimate of $x\left(k^{\prime}\right)$, given all available measurements up until time $k$. Also let $\lambda(A)$ denote the set of eigenvalues to $A$.

\section{PROBLEM FORMULATION}

We consider the problem of controlling the linear process $\mathcal{P}$ over a communication network as in Fig. 1 , where $\mathcal{P}$ is given by the LTI process

$$
x(k+1)=A x(k)+B u(k), x(k) \in \mathbb{R}^{n}, u(k) \in \mathbb{R}^{m} .
$$

The process is controlled by the controller $\mathcal{C}$ which for a sample $x\left(k_{0}\right)$ computes the control signal $u\left(k_{0}\right)$ and sends it to the actuator. The actuator in turn will apply this control signal to the process until it receives a new value from the controller. Jointly with deciding $u\left(k_{0}\right)$ the controller also decides how many discrete time steps $I\left(k_{0}\right)$ it will wait before it needs to change the control signal next time. This value $I\left(k_{0}\right)$ is sent to the network manager which will schedule the sensor to send a new sample at time $k=k_{0}+I\left(k_{0}\right)$. When this sample $x\left(k_{0}+I\left(k_{0}\right)\right)$ arrives to the controller it computes the new control signal $u\left(k_{0}+I\left(k_{0}\right)\right)$ and sends it to the actuator. It also decides on a new back-off time $I\left(k_{0}+I\left(k_{0}\right)\right)$ and sends that to the network manager, which schedules the sensor accordingly. How the scheduling of sensors is done inside the network manager is left outside the scope of this paper.

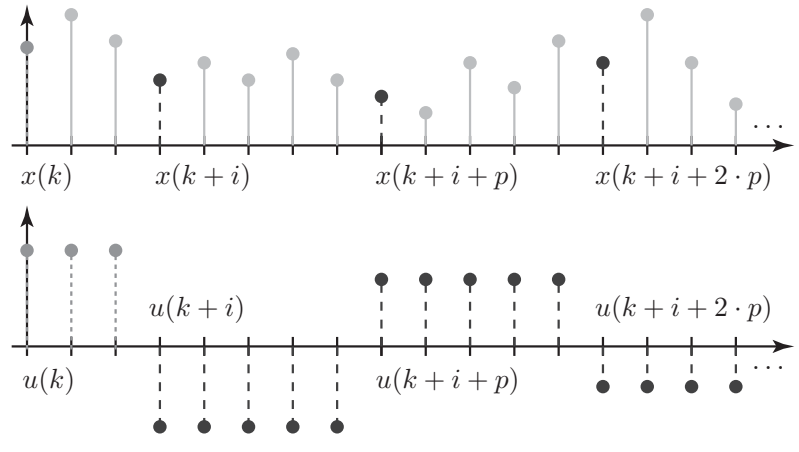

Fig. 2. The prediction horizon. Here typical signal predictions are shown with $I(k)=3$ and $p=5$.

Herein we propose that the controller $\mathcal{C}$ should be implemented as a receding horizon controller which at every sampling instant solves an open-loop optimal control problem by minimizing a cost function $J$, taking both control performance and inter sample time into consideration. The optimizing value of the control signal is then applied to the process for the optimal inter sample time.

\section{OPEN-LOOP OPTIMAL CONTROL PROBLEM}

The open-loop cost function we propose the receding horizon controller $\mathcal{C}$ to minimize at every sampling instant is

$$
J(x(k), i, \mathcal{U})=\frac{\alpha}{i}+\sum_{l=0}^{\infty}\left(\|x(k+l)\|_{Q}^{2}+\|u(k+l)\|_{R}^{2}\right)
$$

where $\alpha \in \mathbb{R}^{+}, Q$ and $R$ are design variables. Further $0<Q$ and $0<R$ are symmetric matrices of appropriate dimensions. We optimize this cost over the constraint that the control sequence $\mathcal{U}=\{u(k), u(k+1), \ldots\}$ should follow the specific shape illustrated in Fig. 2, for some fixed $p \in \mathbb{N}^{+}$. That is, the number of discrete time units $i$ to wait before taking the next sample $x(k+i)$, as well as the levels in the control sequence $\mathcal{U}$ are free variables over which we optimize. Note that neither the state nor the control has a constrained magnitude.

The shape of the control trajectory $\mathcal{U}$ is motivated by the following idea. We will use a slight modification of regular receding horizon control where we at time instant $k$ sample the system and use this sample $x(k)$ to compute the predicted, with respect to the constraints, optimal control trajectory $\mathcal{U}$. We will then apply this sequence from time $k$ until time instant $k+i$ when we will take a new sample $x(k+i)$ and redo the optimization. By this method we get a joint optimization of the control signal to be applied as well as the time to the next sampling instant. By the choice of constraints on the shape of $\mathcal{U}$ we will get the shape of the predicted control signal to coincide with the one we will actually apply, up until time $k+i$. The reason for letting the system be controlled by a control signal with period $p$ after this is that we hope for the receding horizon algorithm to converge to this rate, we will later provide methods for choosing $p$ so that this happens. The reason for wanting convergence to a down sampled control is that we want the system to be sampled at a slow rate when it has reached steady state, at the same time as we want it to be sampled faster in the transients.

Mathematically we may formulate the cost (2) including the constraints as 


$$
\begin{aligned}
& J(x(k), i, \mathcal{U})= \frac{\alpha}{i}+\sum_{l=0}^{i-1}\left(\|x(k+l)\|_{Q}^{2}+\|u(k)\|_{R}^{2}\right) \\
&+\sum_{r=0}^{\infty}\left(\sum _ { l = 0 } ^ { p - 1 } \left(\|x(k+i+r \cdot p+l)\|_{Q}^{2}\right.\right. \\
&\left.\left.+\|u(k+i+r \cdot p)\|_{R}^{2}\right)\right)
\end{aligned}
$$

where the term $\alpha / i$ reflects the cost of sampling. We use this cost to weight the cost of sampling against the classical quadratic control performance cost. For a given $x(k)$, choosing a large $\alpha$ will force $i$ to be larger and hence give longer inter sampling times. By the construction of the cost we may first choose $Q$ and $R$ to get the desired control performance and then tune $\alpha$ to get the desired sampling behavior. One could imagine a more general cost of sampling, here however we found $\alpha / i$ sufficient.

\subsection{Minimization of $J$}

Having defined the open-loop cost (3) we proceed by computing its optimal value. We start by noticing that even though we have a joint optimization problem we may state it as

$$
\underset{i}{\operatorname{minimize}}(\underset{\mathcal{U}}{\operatorname{minimize}} J(x(k), i, \mathcal{U})) .
$$

We will use this separation and start by solving the inner problem, that of minimizing $J(x(k), i, \mathcal{U})$ for a given value of $i$. In order to derive the solution, and for future reference, we need to define some variables.

Definition 1. We define notation for the lifted model as

$$
A_{i}=A^{i}, \quad B_{i}=\sum_{q=0}^{i-1} A^{q} B .
$$

and notation for the generalized weighting matrices as

$$
\begin{aligned}
Q_{i} & =Q_{i-1}+A_{i-1}^{T} Q A_{i-1} \\
R_{i} & =R_{i-1}+B_{i-1}^{T} Q B_{i-1}+R \\
N_{i} & =N_{i-1}+A_{i-1}^{T} Q B_{i-1}
\end{aligned}
$$

where $Q_{1}=Q, R_{1}=R$ and $N_{1}=0$.

Using Definition 1 we may formulate the following

Proposition 1. By straight forward expansion it holds that

$$
\begin{aligned}
& \sum_{l=0}^{i-1}\left(\|x(k+l)\|_{Q}^{2}+\|u(k)\|_{R}^{2}\right)= \\
& =x(k)^{T} Q_{i} x(k)+u(k)^{T} R_{i} u(k)+2 x(k)^{T} N_{i} u(k) .
\end{aligned}
$$

and further that $x(k+i)=A_{i} x(k)+B_{i} u(k)$.

Lemma 1. Assume that $0<Q, 0<R$ and that the pair $\left(A_{p}, B_{p}\right)$ is controllable. Then

$$
\begin{aligned}
\min _{\mathcal{U}} \sum_{r=0}^{\infty}\left(\sum _ { l = 0 } ^ { p - 1 } \left(\|x(k+i+r \cdot p+l)\|_{Q}^{2}\right.\right. & \\
& \left.\left.+\|u(k+i+r \cdot p)\|_{R}^{2}\right)\right)=\|x(k+i)\|_{P_{p}}^{2}
\end{aligned}
$$

where

$$
\begin{aligned}
& P_{p}=Q_{p}+A_{p}^{T} P_{p} A_{p}-\left(A_{p}^{T} P_{p} B_{p}+N_{p}\right) L_{p} \\
& L_{p}=\left(R_{p}+B_{p}^{T} P_{p} B_{p}\right)^{-1}\left(A_{p}^{T} P_{p} B_{p}+N_{p}\right)^{T}
\end{aligned}
$$

and the minimizing control signal is given by

$$
u(k+i+r \cdot p)=-L_{p} x(k+i+r \cdot p) .
$$

Proof. Following Proposition 1 the problem is equivalent to

$$
\begin{aligned}
\min _{\mathcal{U}} \sum_{r=0}^{\infty} & \left(x(k+i+r \cdot p)^{T} Q_{p} x(k+i+r \cdot p)\right. \\
& +u(k+i+r \cdot p)^{T} R_{p} u(k+i+r \cdot p) \\
& \left.+2 x(k+i+r \cdot p)^{T} N_{p} u(k+i+r \cdot p)\right)
\end{aligned}
$$

with

$x(k+i+(r+1) \cdot p)=A_{p} x(k+i+r \cdot p)+B_{p} u(k+i+r \cdot p)$.

This problem has the known optimal solution, see e.g. Bertsekas [1995], $\|x(k+i)\|_{P_{p}}^{2}$. Where $P_{p}$ is given by the Riccati equation (5), which has a solution provided that $0<R_{p}$, implied by $0<R$, and $0<Q_{p}$, implied by $0<Q$, and that the pair $\left(A_{p}, B_{p}\right)$ is controllable. Exactly what is stated in the Lemma.

Using the above results we may formulate the main result of this Section as follows.

Theorem 1. Assume that $0<Q, 0<R$ and that the pair $\left(A_{p}, B_{p}\right)$ is controllable. Then

$$
\min _{\mathcal{U}} J(x(k), i, \mathcal{U})=\frac{\alpha}{i}+\|x(k)\|_{P_{i}}^{2}
$$

where

$$
\begin{aligned}
P_{i} & =Q_{i}+A_{i}^{T} P_{p} A_{i}-\left(A_{i}^{T} P_{i} B_{i}+N_{i}\right) L_{i} \\
L_{i} & =\left(R_{i}+B_{i}^{T} P_{p} B_{i}\right)^{-1}\left(A_{i}^{T} P_{p} B_{i}+N_{i}\right)^{T}
\end{aligned}
$$

and $P_{p}$ is given by (5) in Lemma 1. Denoting the vector of all ones in $\mathbb{R}^{n}$ as $\mathbf{1}_{n}$, the minimizing control signal sequence is given by

$\mathcal{U}=\left\{-L_{i} x(k) \mathbf{1}_{i}^{T},-L_{p} x(k+i+r \cdot p) \mathbf{1}_{p}^{T}, \ldots\right\}, r \in\{0,1, \ldots\}$ where $L_{p}$ also is given by (5) in Lemma 1 .

Proof. From the Theorem we have that $0<Q, 0<R$ and that the pair $\left(A_{p}, B_{p}\right)$ is controllable. Thus we may use Lemma 1 to express the cost (3) as

$$
\begin{aligned}
J(x(k), i, \mathcal{U})=\frac{\alpha}{i}+ & \|x(k+i)\|_{P_{p}}^{2} \\
& +\sum_{l=0}^{i-1}\left(\|x(k+l)\|_{Q}^{2}+\|u(k)\|_{R}^{2}\right) .
\end{aligned}
$$

Now applying Proposition 1 we get

$$
\begin{aligned}
& J(x(k), i, \mathcal{U})=\frac{\alpha}{i}+\|x(k+i)\|_{P_{p}}^{2} \\
& \quad+x(k)^{T} Q_{i} x(k)+u(k)^{T} R_{i} u(k)+2 x(k)^{T} N_{i} u(k) .
\end{aligned}
$$

with $x(k+i)=A_{i} x(k)+B_{i} u(k)$. Minimizing $J(x(k), i, \mathcal{U})$ now becomes a finite horizon optimal control problem with one prediction step into the future. This problem has the well defined solution (6), see e.g. Bertsekas [1995], given by iterating the Riccati equation (7).

Now, getting back to the original problem (4). Provided that the assumptions of Theorem 1 hold, we may apply it giving that

$$
\underset{i, \mathcal{U}}{\operatorname{minimize}} J(x(k), i, \mathcal{U})=\underset{i}{\operatorname{minimize}}\left\{\frac{\alpha}{i}+\|x(k)\|_{P_{i}}^{2}\right\} .
$$

Unfortunately the authors are not aware of any method to solve this problem in general. If however $i$ is restricted to a know finite set $\mathcal{I} \subset \mathbb{N}^{+}$we may find the optimal value within this set for a given value of $x(k)$ by simply evaluating $\frac{\alpha}{i}+\|x(k)\|_{P_{i}}^{2} \forall i \in \mathcal{I}$ and by this finding the $i$ which gives the lowest value of the cost. This need not 


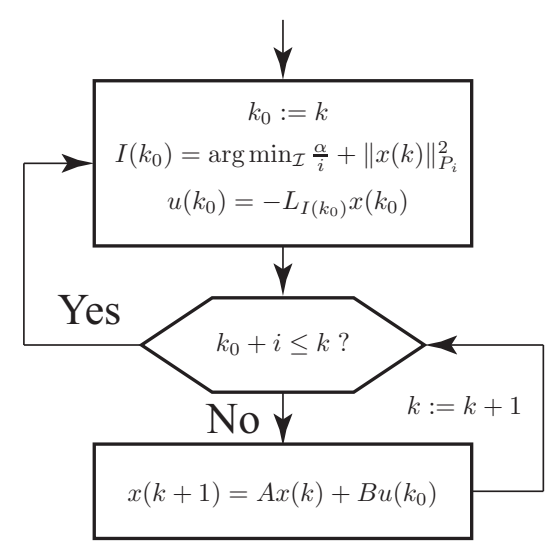

Fig. 3. The proposed receding horizon control algorithm.

however be slow as we may compute $P_{i} \forall i \in \mathcal{I}$ off-line prior to execution.

\section{RECEDING HORIZON IMPLEMENTATION}

We have now defined the open-loop cost which we propose the receding horizon controller to minimize at every sampling instant. Further we have calculated its optimal value as a function of the back-off time $i$ and the corresponding minimizing control signal to be applied. Now we continue with formulating the receding horizon implementation in further detail.

Let the assumptions of Theorem 1 hold and define the set $\mathcal{I} \subset \mathbb{N}^{+}$of allowed values of $i$ and further let $\gamma=\max \mathcal{I}$. From this we may compute $P_{i} \forall i \in \mathcal{I}$ and formulate our proposed control algorithm, illustrated in Fig. 3 and described below.

At time $k=k_{0}$ the state $x(k)$ is sampled giving the sample $x\left(k_{0}\right)$ for which we minimize the cost $\frac{\alpha}{i}+\left\|x\left(k_{0}\right)\right\|_{P_{i}}^{2}$ over $\mathcal{I}$ giving the optimal time to the next sample $I\left(k_{0}\right)$ and the control signal $u\left(k_{0}\right)=-L_{I\left(k_{0}\right)} x\left(k_{0}\right)$ to be applied until then, with $L_{I\left(k_{0}\right)}$ defined as in Theorem 1. Next there is a check if $I\left(k_{0}\right)$ time steps have elapsed since the last sample, if not the control signal $u\left(k_{0}\right)$ is applied to the process $(1)$. After this we again check if $I\left(k_{0}\right)$ time steps have elapsed since the last sample, if not $u\left(k_{0}\right)$ is applied once more. Otherwise a new sample is drawn and a new optimization is performed now starting in $x\left(k_{0}+I\left(k_{0}\right)\right)$.

Remark 1. Even though we are working in uniformly sampled discrete time the state is not sampled at every time instant $k$. Instead the set of samples of the state actually taken is given by the set $\mathcal{D}$, which assuming that the first sample is taken at $k=0$, is given by

$$
\mathcal{D}=\{x(0), x(I(0)), x(I(I(0))), \ldots\} .
$$

\subsection{Stability}

Now having established and detailed our receding horizon control law we continue with giving conditions for when it is stabilizing. First we state the following to handle controllability conditions.

Lemma 2. The system $\left(A_{i}, B_{i}\right)$ is controllable iff the pair $(A, B)$ is controllable and $A$ has no eigenvalue $\lambda(A) \ni \lambda \neq$ 1 such that $\lambda^{i}=1$

Proof. The proof is based on adaptation of Lemma 3.4.1 and Theorem 4 in Sontag [1998] to the problem of down sampling. Let $\mathbf{R}(\cdot)$ be the reachability matrix. For controllability we require

$$
\begin{aligned}
& \mathbf{R}\left(A_{i}, B_{i}\right)=\left[\sum_{q=0}^{i-1} A^{q} B, \ldots, A^{(n-1) i} \sum_{q=0}^{i-1} A^{q} B\right] \\
& =\left(\sum_{q=0}^{i-1} A^{q}\right)\left[B, \ldots, A^{(n-1) i} B\right]=\left(\sum_{q=0}^{i-1} A^{q}\right) \mathbf{R}\left(A_{i}, B\right)
\end{aligned}
$$

to have full rank, i.e., for both $\sum_{q=0}^{i-1} A^{q}$ and $\mathbf{R}\left(A_{i}, B\right)$ to have full rank.

We start by showing the former. According to the Spectral Mapping Theorem the eigenvalues of $f(A)=\sum_{q=0}^{i-1} A^{q}$ will be $f(\lambda)$ with $\lambda \in \lambda(A)$. For $f(A)$ to have full rank we require $f(\lambda) \neq 0, \forall \lambda \in \lambda(A)$. Since

$$
f(1)=i, \quad f(\lambda)=\sum_{q=0}^{i-1} \lambda^{q}=\frac{\lambda^{i}-1}{\lambda-1}, \quad \lambda \neq 1
$$

this is fulfilled iff $\lambda^{i} \neq 1, \lambda(A) \ni \forall \lambda \neq 1$, precisely what is stated in the Lemma.

Moving on, assume that $\mathbf{R}\left(A_{i}, B\right)$ does not have full rank. Which according to the Hautus Lemma in Sontag [1998] is equivalent to that $\left[\lambda^{i} I-A^{i}, B\right]$ is rank deficient, implying $\exists v \neq 0$ such that for some $\lambda$ we have $v^{T}\left[\lambda^{i} I-A^{i}, B\right]=0$ implying $v^{T} \lambda^{i}-v^{T} A^{i}=0$ and $v^{T} B=0$. Thus for $\mathbf{R}\left(A_{i}, B\right)$ to be rank deficient there must exist a left eigenvector $v^{T}$ to $A^{i}$ such that $v^{T} B=0$. Now let $e^{T}$ be a left eigenvector to $A$ which, since $e^{T} A^{i}=e^{T} \lambda^{i}$, also is a left eigenvector to $A^{i}$. Thus for $\mathbf{R}\left(A_{i}, B\right)$ to be rank deficient there must $\exists e$ such that $e^{T} B=0$. Since $\mathbf{R}(A, B)$ has full rank, by the statement in the lemma, we have that for any (non zero) left eigenvector $e^{T}$ it holds that $e^{T}\left[\lambda^{i} I-A^{i}, B\right] \neq 0$ implying $e^{T} B \neq 0$ which contradicts the assumption that $\mathbf{R}\left(A_{i}, B\right)$ is rank deficient.

Using the above and previous results we may now give conditions for when the proposed receding horizon control algorithm is stabilizing.

Theorem 2. Assume $0<Q, 0<R$ and that $(A, B)$ is controllable. If we choose $i \in \mathcal{I} \subset \mathbb{N}^{+}$and $p=p^{*}$ given by

$$
p^{*}=\max \left\{i \mid i \in \mathcal{I}, \lambda^{i} \neq 1 \text { for all } \lambda(A) \ni \lambda \neq 1\right\}
$$

and apply the proposed control algorithm, then

$$
\frac{\alpha}{\gamma} \leq \lim _{k \rightarrow \infty} \min _{i \in \mathcal{I}}\left(\frac{\alpha}{i}+\|x(k)\|_{P_{i}}^{2}\right) \leq \frac{\alpha}{\epsilon}\left(\frac{1}{p^{*}}-(1-\epsilon) \frac{1}{\gamma}\right) .
$$

where $\epsilon$ is the largest value in the interval $(0,1]$ fulfilling

$$
\left(A_{i}-B_{i} L_{i}\right)^{T} P_{p^{*}}\left(A_{i}-B_{i} L_{i}\right) \leq(1-\epsilon) P_{i}, \quad \forall i \in \mathcal{I} .
$$

Proof. Assume that $V_{k}$ is the optimal value of the cost $(3)$ at the current time instant $k$. By assumption $(A, B)$ is controllable. Together with the choice of $p^{*}$ this, via Lemma 2, implies that $\left(A_{p^{*}}, B_{p^{*}}\right)$ is controllable. Defining

$$
\|\hat{x}(k \mid k)\|_{S_{i}}^{2} \triangleq \sum_{l=0}^{i-1}\left(\|\hat{x}(k+l \mid k)\|_{Q}^{2}+\|\hat{u}(k \mid k)\|_{R}^{2}\right)
$$

we may, since by assumption $0<Q$ and $0<R$, use Lemma 1 and Theorem 1 to express $V_{k}$ as 


$$
\begin{aligned}
V_{k} & \triangleq \min _{i \in \mathcal{I}, \hat{u}(k \mid k)} J(x(k), i, \hat{u}(k \mid k)) \\
& =\min _{i \in \mathcal{I}} \frac{\alpha}{i}+\|\hat{x}(k+i \mid k)\|_{P_{p^{*}}}^{2}+\|\hat{x}(k \mid k)\|_{S_{i}}^{2} \\
& =\min _{i \in \mathcal{I}} \frac{\alpha}{i}+\|\hat{x}(k \mid k)\|_{P_{i}}^{2} .
\end{aligned}
$$

Further assume that $V_{k+i}$ is the optimal cost at the next sampling instant $k+i$. Again using Theorem 1 we may express it as

$$
\begin{aligned}
V_{k+i} & \triangleq \min _{j \in \mathcal{I}, \hat{u}(k+i \mid k+i)} J(x(k+i), j, \hat{u}(k+i \mid k+i)) \\
& \leq \min _{\hat{u}(k+i \mid k+i)} J\left(x(k+i), j=p^{*}, \hat{u}(k+i \mid k+i)\right) \\
& =\frac{\alpha}{p^{*}}+\|\hat{x}(k+i \mid k+i)\|_{P_{p^{*}}}^{2}=\frac{\alpha}{p^{*}}+\|\hat{x}(k+i \mid k)\|_{P_{p^{*}}}^{2} .
\end{aligned}
$$

Where the inequality comes from the fact that choosing $j=p^{*}$ is sub-optimal. Taking the difference we get

$$
V_{k+i}-V_{k} \leq \frac{\alpha}{p^{*}}-\frac{\alpha}{i}-\|\hat{x}(k \mid k)\|_{S_{i}}^{2}
$$

which in general is not decreasing. However we may use the following idea to bound this difference. Assume that there $\exists \epsilon \in(0,1]$ and $\beta \in \mathbb{R}^{+}$such that we may write

$$
V_{k+i}-V_{k} \leq-\epsilon V_{k}+\beta
$$

As this should hold for all sampling instances $\mathcal{D}$ given in (8), we have that at $l$ sampling instances into the future, which happens at let's say time $k+l^{\prime}$, we have that

$$
V_{k+l^{\prime}} \leq(1-\epsilon)^{l} \cdot V_{k}+\beta \cdot \sum_{r=0}^{l-i}(1-\epsilon)^{l} .
$$

Since $\epsilon \in(0,1]$ this is equivalent to

$$
V_{k+l^{\prime}} \leq(1-\epsilon)^{l} \cdot V_{k}+\beta \cdot \frac{1-(1-\epsilon)^{l}}{1-(1-\epsilon)},
$$

which as $l \rightarrow \infty$ gives us an upper bound on the cost function, $V_{k+l^{\prime}} \leq \beta / \epsilon$. Applying this idea on our setup we should fulfill

$$
\begin{aligned}
\frac{\alpha}{p^{*}}-\frac{\alpha}{i}- & \|\hat{x}(k \mid k)\|_{S_{i}} \leq \\
& -\epsilon \frac{\alpha}{i}-\epsilon\|\hat{x}(k+i \mid k)\|_{P_{p^{*}}}^{2}-\epsilon\|\hat{x}(k \mid k)\|_{S_{i}}^{2}+\beta .
\end{aligned}
$$

Choosing $\beta=\alpha / p^{*}-(1-\epsilon) \alpha / \gamma$ we have fulfillment if

$$
\epsilon\left(\|\hat{x}(k+i \mid k)\|_{P_{p^{*}}}^{2}+\|\hat{x}(k \mid k)\|_{S_{i}}^{2}\right) \leq\|\hat{x}(k \mid k)\|_{S_{i}}^{2}
$$

Clearly there $\exists \epsilon \in(0,1]$ such that the above relation is fulfilled if $0<\|\hat{x}(k \mid k)\|_{S_{i}}^{2}$. If $\|\hat{x}(k \mid k)\|_{S_{i}}^{2}=0$ we must, following the definition $(10)$ and the assumption $0<Q$, have that $\hat{x}(k \mid k)=0$ and $\hat{x}(k+i \mid k)=0$ and hence the relation is fulfilled also in this case. Using the final step in (11) we may express (12) in easily computable quantities giving the condition

$$
\|\hat{x}(k+i \mid k)\|_{P_{p^{*}}}^{2} \leq(1-\epsilon)\|\hat{x}(k \mid k)\|_{P_{i}}^{2}
$$

As this should hold $\forall x$ and $\forall i \in \mathcal{I}$ we must fulfill

$$
\left(A_{i}-B_{i} L_{i}\right)^{T} P_{p^{*}}\left(A_{i}-B_{i} L_{i}\right) \leq(1-\epsilon) P_{i}, \quad \forall i \in \mathcal{I}
$$

which is stated in the Theorem. Summing up we have

$$
V_{k+l^{\prime}} \leq \frac{\alpha}{\epsilon}\left(\frac{1}{p^{*}}-(1-\epsilon) \frac{1}{\gamma}\right)
$$

which is minimized by maximizing $\epsilon$. From the definition of the cost (2) we may also conclude that $\alpha / \gamma \leq V_{k+l^{\prime}}$. With

$$
V_{k+l^{\prime}}=\min _{i \in \mathcal{I}}\left(\frac{\alpha}{i}+\left\|\hat{x}\left(k+l^{\prime} \mid k+l^{\prime}\right)\right\|_{P_{i}}^{2}\right)
$$

we may conclude that

$$
\frac{\alpha}{\gamma} \leq \lim _{k \rightarrow \infty} \min _{i \in \mathcal{I}}\left(\frac{\alpha}{i}+\|\hat{x}(k \mid k)\|_{P_{i}}^{2}\right) \leq \frac{\alpha}{\epsilon}\left(\frac{1}{p^{*}}-(1-\epsilon) \frac{1}{\gamma}\right) .
$$

Corollary 1. If the assumptions of Theorem 2 hold and further we have that either $p^{*}=\gamma$ or $\alpha=0$, then

$$
\lim _{k \rightarrow \infty} x(k)=0
$$

Proof. From Theorem 2 we have that as $k \rightarrow \infty$

$$
\frac{\alpha}{\gamma} \leq \min _{i \in \mathcal{I}}\left(\frac{\alpha}{i}+\|\hat{x}(k \mid k)\|_{P_{i}}^{2}\right) \leq \frac{\alpha}{\epsilon}\left(\frac{1}{p^{*}}-(1-\epsilon) \frac{1}{\gamma}\right)
$$

which as $p^{*}=\gamma$ simplifies to

$$
\frac{\alpha}{\gamma} \leq \min _{i \in \mathcal{I}}\left(\frac{\alpha}{i}+\|\hat{x}(k \mid k)\|_{P_{i}}^{2}\right) \leq \frac{\alpha}{\gamma}
$$

independent of $\epsilon$. Implying that for the optimal $i$ we have $\|\hat{x}(k \mid k)\|_{P_{i}}^{2}=0$, since $0<P_{i}$ provided $0<Q$ this implies $x(k)=0$ independent of $\alpha$. In the case $\alpha=0$ the bound from Theorem 2 simplifies to that as $k \rightarrow \infty$

$$
0 \leq \min _{i \in \mathcal{I}}\|\hat{x}(k \mid k)\|_{P_{i}}^{2} \leq 0
$$

and hence for the optimal $i$ we have $\|\hat{x}(k \mid k)\|_{P_{i}}^{2}=0$ implying $x(k)=0$ as above.

From the above results we may note the following.

Remark 2. If the assumptions of Theorem 2 hold Corollary 1 will hold except in the extremely rare case that the underlying system $(A, B)$ becomes uncontrollable under down sampling by a factor $\gamma$, see Lemma 2 . If it does not hold one may use Lemma 2 to re-design $\mathcal{I}$ which recovers the case $p^{*}=\gamma$ so that it will hold.

Remark 3. The bound given in Theorem 2 scales linearly with the choice of $\alpha$.

Remark 4. The proposed algorithm guarantees a minimum and a maximum inter sampling time. The minimum time is 1 time step in the time scale of the underlying process (1) and the maximum inter sampling time is $\gamma$ time steps.

\section{NUMERICAL EXAMPLE}

To exemplify the proposed method we will study the single integrator system $\dot{x}=u$ which we discretize using sample and hold with sampling time $T_{s}=1 \mathrm{~s}$ giving us $x(k+$ $1)=x(k)+u(k)$. Since we want the resulting receding horizon control law to be stabilizing we need to make sure that our design fulfills Theorem 2. If we further want it to be asymptotically stabilizing we in addition need it to fulfill the conditions of Corollary 1.

The design procedure is then as follows: First we note that the system $(A, B)=(1,1)$ is controllable. We also note that the system only has the eigenvalue $\lambda=1$ so that (9) in Theorem 2 gives $p^{*}=\gamma$. Hence Corollary 1 will hold for every choice of $\mathcal{I}$ provided that Theorem 2 holds. In essences this means that we may choose the elements in $\mathcal{I}$, i.e. the allowed down sampling rates, freely. A natural way to choose them is to decide on a maximum allowed down sampling rate and then choose $\mathcal{I}$ to contain all rates from 1 up to this number. Let's say that we here want the system to be sampled at least every $5 \cdot T_{s} \mathrm{~s}$, then a good choice is $\mathcal{I}=\{1,2,3,4,5\}$, giving $p^{*}=\gamma=5$. The next step is to decide the weights $0<Q$ and $0<R$ in the quadratic cost function (2). This is done in the same way as in classical Linear Quadratic control, see e.g. Maciejowski [2002]. Here we for simplicity choose $Q=1$ and $R=1$. 
Table 1 . The pre-computed control laws with related cost functions and intermediate variables.

\begin{tabular}{c|cc|ccc|ccc}
$\mathcal{I}$ & $A_{i}$ & $B_{i}$ & $Q_{i}$ & $R_{i}$ & $N_{i}$ & $L_{i}$ & $P_{i}$ & $V_{i}(x)$ \\
\hline 1 & 1 & 1 & 1 & 1 & 0 & 0.70 & 1.70 & $\alpha / 1+P_{1} \cdot x^{2}$ \\
2 & 1 & 2 & 2 & 3 & 1 & 0.46 & 1.73 & $\alpha / 2+P_{2} \cdot x^{2}$ \\
3 & 1 & 3 & 3 & 8 & 3 & 0.35 & 1.89 & $\alpha / 3+P_{3} \cdot x^{2}$ \\
4 & 1 & 4 & 4 & 18 & 6 & 0.28 & 2.08 & $\alpha / 4+P_{4} \cdot x^{2}$ \\
$5=p^{*}$ & 1 & 5 & 5 & 35 & 10 & 0.23 & 2.30 & $\alpha / 5+P_{5} \cdot x^{2}$
\end{tabular}

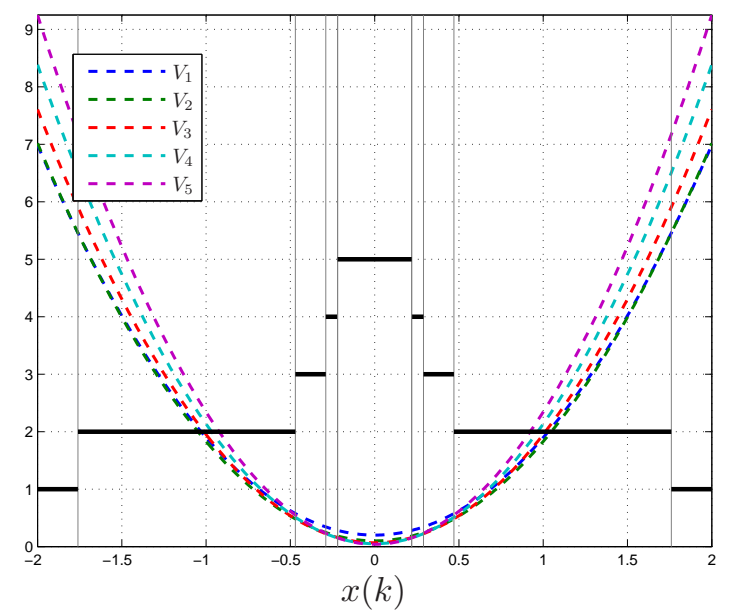

Fig. 4. The cost functions $V_{i}(x(k))$ (dashed) together with the partitioning of the state space and the back-off time $I(k)=\arg \min _{\mathcal{I}} V_{i}(x(k))$ (solid).

Now having guaranteed that the conditions of Theorem 2 and Corollary 1 hold we have also guaranteed that the conditions of Theorem 1 are fulfilled. Hence we may use it to compute the needed state feedback gains and cost function matrices that are used by the receding horizon controller. The results from these computations are shown in Table 1, together with some of the intermediate variables from Definition 1 . Here we see that the cost functions are quadratic functions in the state $x$ where the coefficient $P_{i}$ are functions of $Q$ and $R$. We also see that the cost to sample $\alpha / i$ enters linearly and as we change it we will change the offset level of the curves and thereby their values related to each other. However it will not affect the state feedback gains.

A graphical illustration of the cost functions in Table 1, for the choice $\alpha=0.2$, is shown in Fig. 4 together with the curve $I(k)=\arg \min _{\mathcal{I}} V_{i}(x(k))$, i.e. the index of the cost function which has the lowest value for a given state $x(k)$. This is the partitioning of the state space that the receding horizon controller will use to choose which of the state feedback gains to apply and how long to wait before sampling again. That is: If a sample is taken with the value $x=1$ we may using Fig. 4 conclude that the control cost (3) is minimized by applying the control $u=-L_{2} x=-0.46 \cdot 1$ for the next $2 \cdot T_{s} \mathrm{~s}$ before taking a new sample and reevaluating the receding horizon control law.

Applying our receding horizon control law described in Fig. 3 using the results in Table 1 to our integrator system when initialized in $x(0)=2$ we get the response shown in Fig. 5(a). As the first sample is taken at $x(0)=2$ we see from Fig. 4 that the control cost is minimized by applying $u=-L_{1} x(0)$ for $1 \cdot T_{s} \mathrm{~s}$ before taking a new sample. At this next sample $x(1)=0.6$ we may, again using Fig. 4, conclude that the cost now is minimized by applying $u=-L_{2} x(1)$ for $2 \cdot T_{s} \mathrm{~s}$ before taking a new sample. This procedure is then repeated for the following samples. Note here that the system will converge to the fixed sampling rate $p^{*}$ as the state converges.

It may now appear as it is sufficient to use periodic control and sample the system every $p^{*} \cdot T_{s} \mathrm{~s}$ to get good control performance. To compare the performance of this sampling strategy with the one above we apply the control which minimizes the same cost function (2) as above with the exception that the system now may only be sampled every $p^{*} \cdot T_{s} \mathrm{~s}$. This is in fact the same as using the receding horizon control above while restricting the controller to choose $i=p^{*}$ every time. The resulting simulations are shown in Fig. 5(b). As seen there is a large degradation of the performance in the transient while the stationary behavior is almost the same. By this we can conclude that it is not sufficient to sample the system every $p^{*} \cdot T_{s} \mathrm{~s}$ if we want to achieve the same transient performance as with the adaptive sampling.

In the initial transient response the receding horizon controller sampled after one time instant. This indicates that there is performance to gain by sampling every time instant. To investigate this we apply the control which minimizes the same cost function (2), now with the exception that the system may be sampled every $T_{s}$ s, i.e. classical unconstrained Linear Quadratic control. Now simulating the system we get the response shown in Fig. 5(c). As expected we get slightly better transient performance in this case compared to the adaptive sampling scheme, it is however comparable. Note however that this improvement comes at the cost of a drastically increased communication need, which may not be suitable for systems where multiple loops share the same wireless medium.

From the above we may conclude that our adaptive method combines the low communication rate in stationarity of the slow periodic controller with the quick transient response of the fast periodic sampling. In fact we may, using our adaptive method, in this example recover the transient behavior of fast periodic sampling at the communication cost of one extra sample compared to slow periodic sampling. The reason for this is that the fast sampling rate only is needed in the transient while we in stationarity can obtain sufficient performance with a lower rate.

\section{CONCLUSIONS AND FUTURE WORK}

We have presented a method which at every sampling instant computes the optimal control signal to be applied as well as the optimal time to wait before taking the next sample. It is shown that this control law may be realized using MPC and computed explicitly. The controller is also shown to be stabilizing under mild assumptions. Simulation results show that the use of the presented control law may help reducing the required amount of communication without almost any loss of performance compared to fast periodic sampling.

An interesting topic for future research is to study the influence of noise. It would tractable to extend the approach to handle adaptive sampling of multiple loops communicating over a shared network, while guaranteeing a feasible schedule. Further extending the framework to handle constrained and non-linear systems is natural.

\section{REFERENCES}

A. Anta and P. Tabuada. To sample or not to sample: Selftriggered control for nonlinear systems. IEEE Transac- 

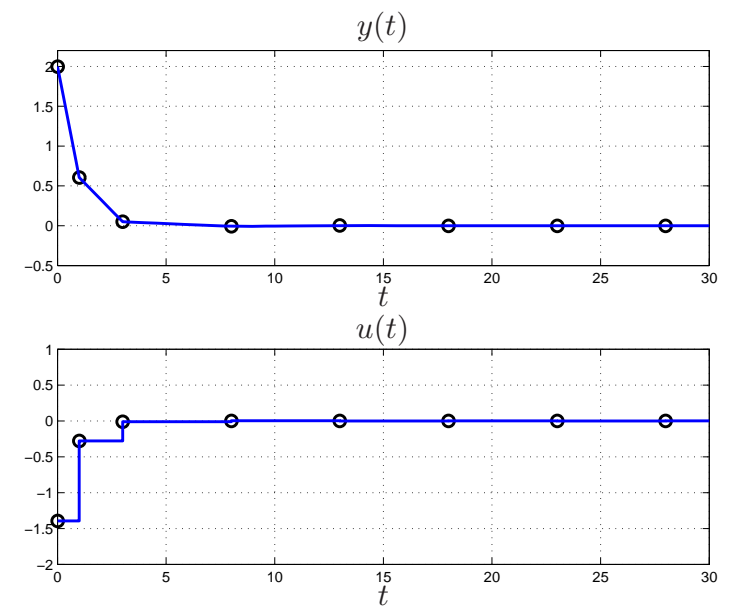

(a) System response of the integrator system when minimizing the cost by adaptively choosing the sampling time.
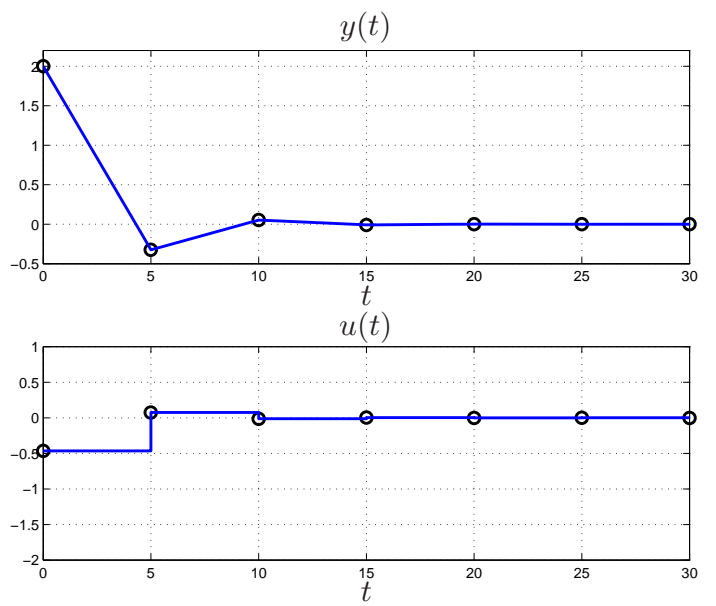

(b) System response of the integrator system when minimizing the cost by sampling every $5^{\text {th }}$ second.
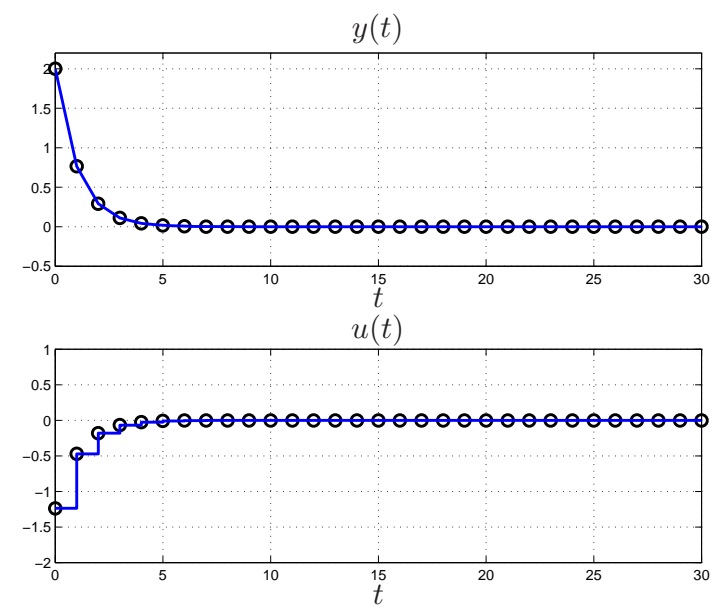

(c) System response of the integrator system when minimizing the cost by sampling every second.

Fig. 5. Comparing control performance for different sampling policies. tions on Automatic Control, 55(9):2030 -2042, 2010.

P. Antsaklis and J. Baillieul. Special issue on technology of networked control systems. Proceedings of the IEEE, 95(1), 2007.

K-E. Årzén. A simple event-based PID controller. Preprints 14th World Congress of IFAC. Beijing, China, 1999.

K.J. Åström and B Bernhardsson. Comparison of periodic and event based sampling for first-order stochastic systems. In Preprints 14th World Congress of IFAC, Beijing, P.R. China, 1999.

D. Bernardini and A. Bemporad. Energy-aware robust model predictive control based on noisy wireless sensors. Automatica, 2011. In press.

D. P. Bertsekas. Dynamic Programming and Optimal Control, volume 1. Athena Scientific, 1995.

A. Casavola, E. Mosca, and M. Papini. Predictive teleoperation of constrained dynamic systems via internetlike channels. IEEE Transactions on Control Systems Technology, 14(4):681 -694, 2006.

A. Eqtami, D.V. Dimarogonas, and K.J. Kyriakopoulos. Event-triggered control for discrete-time systems. Proccedings of American Control Conference, 2010.

W.P.M.H. Heemels, J.H. Sandee, and P.P.J. van den Bosch. Analysis of event-driven controllers for linear systems. International Journal of Control, pages 81(4), 571-590, 2008.

G. P. Liu, D. Rees J. X. Mu, and S. C. Chai. Design and stability analysis of networked control systems with random communication time delay using the modified MPC. International Journal of Control, 79:288 -297, 2006.

J. Liu, D. Muñoz de la Peña, and P. D. Christofides. Distributed model predictive control of nonlinear systems subject to asynchronous and delayed measurements. Automatica, 46:52 - 61, 2010.

J. M. Maciejowski. Predictive Control with Constraints. Prentice Hall, 2002.

M. Mazo Jr., A. Anta, and P. Tabuada. An ISS selftriggered implementation of linear controllers. Automatica, 46(8):1310-1314, 2010.

E. D. Sontag. Mathematical Control Theory. Deterministic Finite-Dimensional Systems, volume 6 of Texts in Applied Mathematics. Springer-Verlag, New York, second edition, 1998

P. Tabuada. Event-triggered real-time scheduling of stabilizing control tasks. IEEE Transactions on Automatic Control, 52(9):1680-1685, 2007.

P. L. Tang and C. W. de Silva. Compensation for transmission delays in an ethernet-based control network using variable-horizon predictive control. IEEE Transactions on Control Systems Technology, 14(4):707 -718, 2006.

M. Velasco, J. Fuertes, and P. Martí. The self triggered task model for real-time control systems. 24th IEEE Real-Time Systems Symposium (work in progress), pages 67-70, 2003.

$\mathrm{X}$. Wang and M. Lemmon. Self-triggered feedback control systems with finite-gain 12 stability. IEEE Transactions on Automatic Control, 45:452-467, 2009.

A. Willig. Recent and emerging topics in wireless industrial communications: A selection. IEEE Transactions on Industrial Informatics, 4(2):102 -124, May 2008. ISSN 1551-3203. doi: 10.1109/TII.2008.923194.

Y.-B. Zhao, G. P. Liu, and D. Rees. Improved predictive control approach to networked control systems. IET Control Theory and Applications, 2(8):675 -681, 2008. 